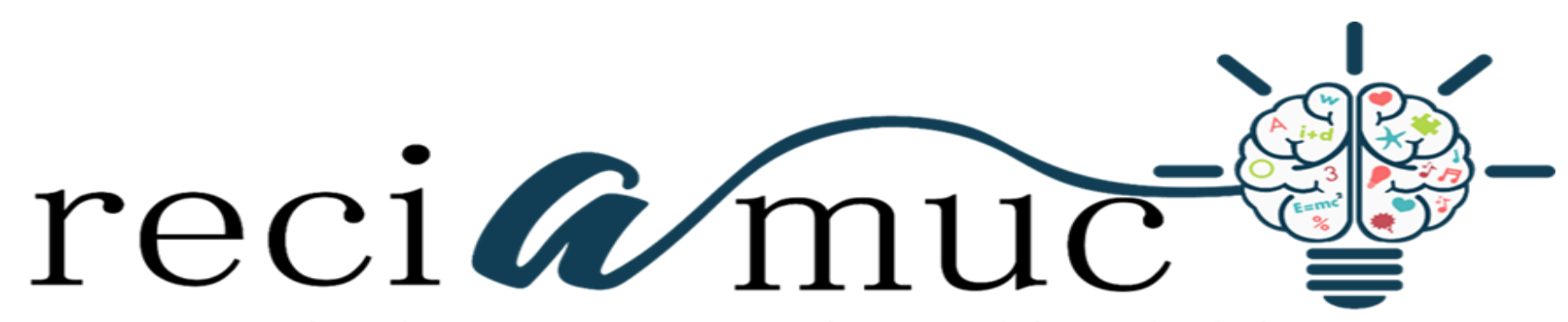

Revista cientifica de investigación actualización del mundo de las ciencias

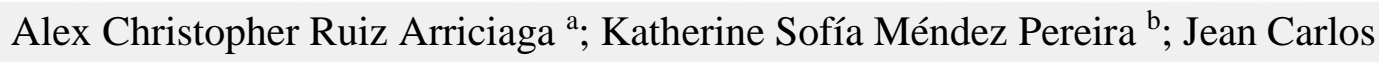
Torres Fernández ${ }^{c}$

Diagnóstico diferencial del absceso hepático amebiano Differential diagnosis of amoebic liver abscess

Revista Científica de Investigación actualización del mundo de las Ciencias. Vol. 3 núm., 4, octubre: 2588-0748, 2019, pp. 76-92

DOI: $10.26820 /$ reciamис/3.(4).octubre.2019.76-92

URL: http://reciamuc.com/index.php/RECIAMUC/article/view/390

Código UNESCO: 3205 Medicina Interna

Tipo de Investigación: Artículo de Revisión

(C) RECIAMUC; Editorial Saberes del Conocimiento, 2019

Recibido: $15 / 05 / 2019$

Aceptado: 07/08/2019

Publicado: 01/10/2019

Correspondencia: alexruiza3@gmail.com
a. Médico; Investigador Independiente; Guayaquil, Ecuador; alexruiza3@gmail.com
b. Médico; Investigador Independiente; Guayaquil, Ecuador; kath_mendez@ hotmail.com
c. Médico; Investigador Independiente; Guayaquil, Ecuador; jecacio-107@ hotmail.es 


\section{Diagnóstico diferencial del absceso hepático amebiano}

Vol. 3, núm. 4., (2019)

Alex Christopher Ruiz Arriciaga; Katherine Sofía Méndez Pereira;

Jean Carlos Torres Fernández

\section{RESUMEN}

El absceso hepático amebiano en todos los casos es secundario a la colonización e invasión de la pared del intestino grueso por la E. histolytica. El absceso hepático amebiano se presenta con más frecuencia en el lóbulo derecho porque recibe la mayor parte del drenaje venoso del ciego y del colon ascendente, donde la amebiasis intestinal se desarrolla usualmente. Pueden ser únicos o múltiples y su tamaño es variable. Es una patología aguda o subaguda, que se presenta en pacientes de mediana edad, sin preferencia de género, cuya sintomatología principal es fiebre y dolor en hipocondrio derecho, con la colelitiasis como principal antecedente patológico. El Absceso Hepático es una colección de pus rodeado de cápsula fibrosa que, según su etiología, se pueden dividir en dos grupos: piógenos (es una zona llena de pus en el hígado) y amebianos (es la principal complicación extra intestinal de la infección por este protozoario E. histolytica). Como herramienta para el diagnóstico existen diversas alternativas; los estudios imaginológicos como la radiografía de tórax, ecografía hepática y de vías biliares, la tomografía computarizada (TAC) y la resonancia magnética (RM). La determinación de anticuerpos en diferentes fluidos corporales como suero o saliva y de antígeno en el fluido del drenaje percutáneo del absceso hepático. Adicionalmente, los estudios endoscópicos, como la rectosigmoidoscopia. El fármaco más utilizado para tratar el absceso hepático amebiano es el metronidazol 500 a 750 mg por vía oral o intravenosa, tres veces al día durante 7 a 10 días. Se iniciará tratamiento ante la sospecha de absceso hepático amebiano, se indicará punción percutánea si el paciente presenta: persistencia de síntomas clínicos. El drenaje laparoscópico combinado con antibiótico terapia es una alternativa quirúrgica en paciente seleccionado o posterior a fracaso de drenaje percutáneo. La metodología usada es descriptiva, con un enfoque documental, es decir, revisar fuentes disponibles en la red, como google académico, con contenido oportuno y relevante desde el punto de vista científico que enriquezca el análisis del tema planteado en este artículo.

Palabras claves: Entamoeba Histolytica; Absceso Hepático Amebiano; Absceso Piógeno; Metronidazol; Punción; Pruebas ELIZA. 


\section{Diagnóstico diferencial del absceso hepático amebiano}

Vol. 3, núm. 4., (2019)

Alex Christopher Ruiz Arriciaga; Katherine Sofía Méndez Pereira;

Jean Carlos Torres Fernández

\section{ABSTRACT}

Amebic liver abscess in all cases is secondary to colonization and invasion of the wall of the large intestine by E. histolytica. Amebic liver abscess occurs most frequently in the right lobe because it receives most of the venous drainage of the cecum and ascending colon, where intestinal amebiasis usually develops. They can be single or multiple and their size is variable. It is an acute or subacute pathology, which occurs in middle-aged patients, without gender preference, whose main symptomatology is fever and pain in the right hypochondrium, with cholelithiasis as the main pathological antecedent. The Liver Abscess is a collection of pus surrounded by a fibrous capsule that, according to its etiology, can be divided into two groups: pyogens (it is an area full of pus in the liver) and amebic (it is the main extra intestinal complication of infection due to this protozoan E. histolytica). As a tool for diagnosis there are several alternatives; Imaging studies such as chest radiography, liver and bile duct ultrasound, computed tomography (CT) and magnetic resonance imaging (MRI). The determination of antibodies in different body fluids such as serum or saliva and antigen in the percutaneous drainage fluid of the liver abscess. Additionally, endoscopic studies, such as rectosigmoidoscopy. The most commonly used drug to treat amoebic liver abscess is metronidazole 500 to $750 \mathrm{mg}$ orally or intravenously, three times a day for 7 to 10 days. Treatment will be initiated on suspicion of amoebic liver abscess, percutaneous puncture will be indicated if the patient presents: persistence of clinical symptoms. Laparoscopic drainage combined with antibiotic therapy is a surgical alternative in a selected patient or after percutaneous drainage failure. The methodology used is descriptive, with a documentary approach, that is, reviewing sources available on the web, such as google scholar, with timely and scientifically relevant content that enriches the analysis of the topic raised in this article.

Key words: Entamoeba Histolytica; Amebic Hepatic Abscess; Pyogenic Abscess; Metronidazole; Puncture; ELIZA tests. 


\section{Diagnóstico diferencial del absceso hepático amebiano}

Vol. 3, núm. 4., (2019)

Alex Christopher Ruiz Arriciaga; Katherine Sofía Méndez Pereira;

Jean Carlos Torres Fernández

\section{Introducción.}

El dolor abdominal es un síntoma que caracteriza a muchas enfermedades. Cuando este se acompaña de fiebre, se determina si la causa de estos síntomas, es infecciosa o no. En caso de ser infecciosa, se debe investigar el agente causal de las mismas. Una de estas enfermedades es el absceso hepático, que se caracteriza por algunos síntomas constitucionales, que si no se tratan a tiempo, los pacientes pueden tener muchas complicaciones, o incluso la muerte.

La Entamoeba histolytica es el protozoario encargado de producir la amibiasis. Posee diferentes formas: quiste, trofozoito y pre quiste, siendo las formas infectante, invasora de tejido intestinal y la de transición respectivamente. Los portadores de los quistes de E. histolytica son la fuente de infección, transmitiéndola vía oral por medio de alimentos, agua, manos y moscas. El pH del estómago debilita los quistes y en el intestino delgado terminan de romperse, originando trofozoitos los cuales invaden tejidos o se albergan sobre las criptas.

Puede irrumpir hacia otros órganos, como el hígado; es la localización amebiana más frecuente después del colon, los trofozoítos penetran el intestino grueso y por vía porta llegan al hígado donde producen puntos de necrosis debido a trombos en pequeños vasos formando el absceso. Su diagnóstico diferencial incluye: absceso piogénico, hidatidosis hepatoma y el cáncer metastásico. El absceso hepático amebiano es más común que el absceso piogénico pero tienen las mismas características ecográficas.

Es una patología relacionada directamente con las malas condiciones sanitarias, su incidencia disminuye con medidas que tiendan a mejorar estas condiciones. Es importante como se explicará durante el desarrollo de este artículo, diferenciarlo de otras patologías similares 


\section{Diagnóstico diferencial del absceso hepático amebiano}

Vol. 3, núm. 4., (2019)

Alex Christopher Ruiz Arriciaga; Katherine Sofía Méndez Pereira;

Jean Carlos Torres Fernández

mediante un diagnostico asertivo, haciendo uso de todas las herramientas actualizadas en cuanto a laboratorio, imágenes y demás medios disponibles al alcance de la ciencia médica en procura de la mejora del paciente.

\section{Metodología.}

Esta investigación está dirigida al estudio del "Diagnóstico diferencial del absceso hepático amebiano”. Para realizarlo se usó una metodología tipo descriptiva, con un enfoque documental, es decir, revisar fuentes disponibles en la red, como google académico, con contenido oportuno y relevante desde el punto de vista científico para dar respuesta a lo tratado en el presente artículo y que sirvan de inspiración para realizar otros proyectos. Las mismas pueden ser consultadas al final, en la bibliografía.

\section{Resultados.}

El absceso hepático amebiano (AHA) en todos los casos es secundario a la colonización e invasión de la pared del intestino grueso por la E. histolytica, aún en los casos en que no es posible demostrar antecedentes clínicos de disentería, ni la presencia del parásito en los estudios coprológicos o lesiones en el material de autopsia (Marin, Octubre 2000). Para la invasión hepática se han postulado tres vías:

- Supone que el parásito tiene la capacidad de traspasar la pared intestinal alcanzando la cavidad peritoneal y luego el hígado, lo cual no se ha demostrado en humanos.

- Propone la diseminación linfática pero en raras ocasiones se encuentran trofozoitos en los vasos linfáticos. 


\section{Diagnóstico diferencial del absceso hepático amebiano}

Vol. 3, núm. 4., (2019)

Alex Christopher Ruiz Arriciaga; Katherine Sofía Méndez Pereira;

Jean Carlos Torres Fernández

- Indica que el parásito invade la mucosa colónica, forma úlceras y penetra a las vénulas submucosas abordando el sistema porta para luego alcanzar el hígado.

La mayoría de trofozoitos sean lisados en el hígado y sólo permanecen cuando llegan en numerosas cantidades, tienen mayor virulencia o existen factores predisponentes como disminución de defensas, desnutrición, enfermedades concomitantes o alcoholismo. Cuando los trofozoitos sobreviven forman trombosis a nivel de los sinusoides con infartos locales, necrosis y licuefacción originando micro abscesos que pueden progresar y confluir. Cuando el absceso está bien formado tiene cápsula fibrosa con paredes de parénquima comprimido donde pueden encontrarse trofozoitos, puesto que en el contenido es infrecuente su hallazgo.

El término absceso en esta etapa se considera inexacto, ya que no hay pus neutrófilos en la cavidad, sino detritus proteináceos acelulares. Dentro de los mecanismos del parásito implicados en la invasión y destrucción tisular se han establecido: la adhesión de los trofozoitos a las células epiteliales, citólisis dependiente de contacto, liberación de proteasas y otras enzimas degradativas, fagocitosis y resistencia del parásito a los mecanismos de defensa del huésped. Diversos autores reconocen que el AHA consta de dos fases bien definidas: pre-supurativa y supurativa. Se inicia con la invasión del trofozoito y continúa hasta la producción de micro abscesos que marcan el origen de la segunda fase de absceso propiamente dicho. La fase pre-supurativa fue considerada por mucho tiempo como "hepatitis amebiana.

El absceso hepático amebiano se presenta con más frecuencia en el lóbulo derecho porque recibe la mayor parte del drenaje venoso del ciego y del colon ascendente, donde la amebiasis intestinal se desarrolla usualmente. Pueden ser únicos o múltiples y su tamaño es variable desde 


\section{Diagnóstico diferencial del absceso hepático amebiano}

Vol. 3, núm. 4., (2019)

Alex Christopher Ruiz Arriciaga; Katherine Sofía Méndez Pereira;

Jean Carlos Torres Fernández

unos pocos milímetros hasta lesiones de $16-18 \mathrm{~cm}$ que pueden reemplazar hasta $90 \%$ del hígado normal (Viasus, 2004). Dada la alta frecuencia en el lóbulo derecho en los segmentos vecinos al hemi diafragma la reacción pleural por vecindad es usual.

El AH es una patología aguda o subaguda, que se presenta en pacientes de mediana edad, sin preferencia de género, cuya sintomatología principal es fiebre y dolor en hipocondrio derecho, con la colelitiasis como principal antecedente patológico. Es importante desarrollar protocolos de manejo de esta enfermedad, con el propósito de mejorar la calidad de la información consignada en las historias clínicas, dado que esto representa una clara limitación a la hora de realizar el diagnóstico (Bernejo, 2014). La tabla $\mathrm{N}^{\circ} 1$ resume los síntomas y signos del AHA.

Tabla 1. Principales síntomas y signos del absceso hepático amebiano

\begin{tabular}{|l|l|}
\hline Síntomas & Signos \\
\hline Localización lóbulo derecho. & Hepatomegalia dolorosa. \\
Dolor en hipocondrio derecho. & Taquicardia, taquipnea y fiebre. \\
$\begin{array}{l}\text { Fiebre. } \\
\text { Síntomas inespecíficos: malestar general, } \\
\text { anorexia, adinamia, pérdida de peso, }\end{array}$ & Disminución del murmullo vesicular. \\
palidez. & Derrame pleural. \\
\hline
\end{tabular}




\section{Diagnóstico diferencial del absceso hepático amebiano}

Vol. 3, núm. 4., (2019)

Alex Christopher Ruiz Arriciaga; Katherine Sofía Méndez Pereira;

Jean Carlos Torres Fernández

\section{Dolor pleurítico.}

Fuente: (Marin, Octubre 2000)

El absceso hepático amebiano es causado por la Entamoeba histolytica. Este parásito causa la amibiasis, una infección intestinal también llamada disentería amebiana. Después de que ha ocurrido una infección, el parásito puede ser transportado a través de la sangre desde los intestinos hasta el hígado. La amebiasis se disemina por consumir agua o alimentos contaminados con materia fecal. En algunas ocasiones, esto se debe al uso de excrementos humanos como fertilizantes. La amebiasis también se disemina por del contacto directo entre personas.

La infección se presenta a nivel mundial, pero es más común en las áreas tropicales donde hay situaciones de hacinamiento y malas condiciones sanitarias. África, Latinoamérica, el sudeste asiático y la India tienen problemas de salud considerables a raíz de esta enfermedad. Los factores de riesgo que predisponen al absceso hepático amebiano son, entre otros (Feldman, 2017):

- Viaje reciente a una región tropical.

- Alcoholismo.

- Cáncer.

- Inmunodepresión, incluso infección por VIH/SIDA.

- Desnutrición.

- Edad avanzada.

- Embarazo.

- Uso de esteroides. 


\section{Diagnóstico diferencial del absceso hepático amebiano}

Vol. 3, núm. 4., (2019)

Alex Christopher Ruiz Arriciaga; Katherine Sofía Méndez Pereira;

Jean Carlos Torres Fernández

En los abscesos hepáticos se puede encontrar leucocitosis asociado a anemia y velocidad de sedimentación incrementada. Dentro del perfil hepático la fosfatasa alcalina, bilirrubinas y transaminasas se encuentran usualmente aumentadas y se evidencia presencia de hipoalbuminemia; nuestros resultados mostraron mayor frecuencia de hipoalbuminemia que otros estudios, lo que se ha descrito asociado a absceso piógeno. En el AH piógeno, el tratamiento antibiótico empírico de amplio espectro vía parenteral debe ser suministrado tan pronto como se sospeche el diagnóstico.

Estos antibióticos incluyen penicilina, un aminoglucósido, y metronidazol, los cuales son efectivos contra E coli, K pneumoniae, bacteroides enterococos, y estreptococos anaerobios. El régimen antibiótico establecido puede variar según el resultado de los cultivos. El tratamiento debe durar entre dos a cuatro semanas, dependiendo del número de abscesos, la mejoría clínica y el resultado de los cultivos realizados. En las últimas décadas, se han introducido nuevas opciones de diagnóstico y tratamiento no quirúrgico, el manejo debe incluir el drenaje del absceso, las técnicas de drenaje incluyen drenaje percutáneo guiado por ecografía o TC.

El Absceso Hepático (AH) es una colección de pus rodeado de cápsula fibrosa que, según su etiología, se pueden dividir en dos grupos: piógenos y amebianos. El absceso hepático amebiano frecuentemente se presenta como fiebre sin foco ( $\sin$ hepatomegalia sensible y sin ictericia).

Esta enfermedad debe diferenciarse del absceso hepático piógeno, que tiene un manejo diferente. Algunas características clínicas que permiten su diferenciación se encuentran en la tabla 2. Pueden encontrarse complicaciones en una tercera parte de los casos, las más comunes son derrame pleural, peritonitis y abscesos sub frénicos que se encuentran en un $20 \%, 10 \%$ y $7 \%$ de 


\section{Diagnóstico diferencial del absceso hepático amebiano}

Vol. 3, núm. 4., (2019)

Alex Christopher Ruiz Arriciaga; Katherine Sofía Méndez Pereira;

Jean Carlos Torres Fernández

los casos. Otras complicaciones, como empiema y abscesos pulmonares son realmente raros. La tabla presentada a continuación compara el absceso amebiano versus el absceso piógeno.

Tabla 2. Diferencias clínicas entre el absceso hepático amebiano y el absceso piógeno.

\begin{tabular}{|l|l|l|}
\hline Característica & Amebiano & \multicolumn{1}{|c|}{ Mí́geno de 50 } \\
\hline Edad & Menos de 40 & $30-40 \%$ \\
\hline Dolor en el hipocondrio derecho & $71-100 \%$ & $95-100 \%$ \\
\hline Fiebre & $95-100 \%$ & $75-80 \%$ \\
\hline Escalofrío & Menos del 30\% & Menos del 5\% \\
\hline Serología para la E. Histolytica & $98-100 \%$ & $50 \%$ solitario \\
\hline Absceso & Más de 95\% solitario & Lóbulo derecho \\
\hline Ubicación & Lóbulo derecho & Frecuente \\
\hline Prurito, ictericia, AST elevadas & Poco frecuente & Frecuente \\
\hline Fosfatasa alcalina elevada & Frecuente & Sí \\
\hline Hemocultivo (+) & No el 10-15\% & \\
\hline Mortalidad & Menos del 5\% & (a) \\
\hline
\end{tabular}

Fuente: (Pereira, Marzo de 2014) 


\section{Diagnóstico diferencial del absceso hepático amebiano}

Vol. 3, núm. 4., (2019)

Alex Christopher Ruiz Arriciaga; Katherine Sofía Méndez Pereira;

Jean Carlos Torres Fernández

\section{Diagnóstico.}

Como herramienta para el diagnóstico de amibiasis extra intestinal o absceso hepático amebiano existen diversas alternativas; los estudios imaginológicos como la radiografía de tórax, ecografía hepática y de vías biliares, la tomografía computarizada (TAC) y la resonancia magnética (RM). La determinación de anticuerpos en diferentes fluidos corporales como suero o saliva y de antígeno en el fluido del drenaje percutáneo del absceso hepático. Adicionalmente, los estudios endoscópicos, como la rectosigmoidoscopia para visualizar las úlceras en botón de camisa, de donde se debe tomar la biopsia para la búsqueda de los trofozoítos, realizar el diagnóstico diferencial con otras patologías del colon especialmente cuando la diarrea persiste a pesar del diagnóstico y tratamiento adecuado y en pacientes inmunocomprometidos por el riesgo de infección oportunista (López, 2008).

A todo paciente con cuadro clínico sugestivo de amebiasis extra intestinal se debe solicitar un cuadro hemático que puede revelar leucocitosis con neutro filia y elevación de velocidad de sedimentación globular (VSG); las enzimas hepáticas (alanina-aminotransferasa, aspartato aminotransferasa, fosfatasa alcalina y tiempo de protrombina pueden estar aumentados con disminución de la albúmina sérica pero no permiten discriminar la etiología.

El diagnóstico del absceso hepático amebiano se basa en la presencia de serología positiva asociada a hallazgos imagenológicos compatibles. En la tomografía abdominal puede encontrarse una lesión hipo densa bien definida que tiene preferencia sobre el lóbulo derecho del hígado. En la ecografía el absceso hepático aparece como una masa hipo ecóica bien definida con una pared delgada y algunas veces puede tener aire en su interior. Se ha mencionado la clasificación de 


\section{Diagnóstico diferencial del absceso hepático amebiano}

Vol. 3, núm. 4., (2019)

Alex Christopher Ruiz Arriciaga; Katherine Sofía Méndez Pereira;

Jean Carlos Torres Fernández

N'Gbesso para el reporte de los hallazgos ecográficos que se correlaciona bien con el manejo del paciente (Pereira, Marzo de 2014).

En el examen general de heces, la presencia de quistes de Entamoeba histolytica aumenta la sospecha de causa amebiana pero no confirma el diagnóstico. En la radiografía de tórax, puede haber elevación del diafragma derecho a expensas de la hepatomegalia. El ultrasonido abdominal es el método más utilizado para diagnóstico, debido a su bajo costo y fácil acceso, con un $90 \%$ de asertividad. También, puede describir el tamaño, cantidad y posición exacta del absceso. También, diferencia de un tumor sólido o enfermedad de vía biliar. Y este, se observa como una masa redonda u ovalada, hipo ecoica con márgenes definidos. Por otra parte, la tomografía axial computarizada abdominal tiene una sensibilidad de $95 \%$, pero debido a su alto costo, se solicita como último recurso cuando existe mucha duda en el diagnóstico (Girón, Diciembre 2011).

Los procedimientos serológicos más empleados para la determinación de amebiasis extra intestinal son: inmuno difusión (ID), contra inmuno electroforesis (CIE), hemoaglutinación indirecta (HAI) y ELISA. La inmuno difusión es una reacción de antígeno - anticuerpo, se considerará como positiva la presencia de una banda de precipitación. La CIE fue una prueba empleada desde la década de los años setenta, antes del advenimiento de la ELISA. La HAI con efectividad alrededor de 99\% y sensibilidad cercana a 93\%, tiene la particularidad de ser rápida y económica, lo que la ha convertido, al igual que la técnica de ELISA, en candidata para ser utilizada como método rutinario.

Enzyme - Linked Immunosorbent Assay (ELISA) para anticuerpos IgG: es una reacción antígeno - anticuerpo. Se puede realizar determinación de diferentes tipos de anticuerpos 


\section{Diagnóstico diferencial del absceso hepático amebiano}

Vol. 3, núm. 4., (2019)

Alex Christopher Ruiz Arriciaga; Katherine Sofía Méndez Pereira;

Jean Carlos Torres Fernández

específicos IgG, IgM e IgA. La prueba de ELISA IgG contra E. Histolytica. Para el diagnóstico de absceso hepático amebiano es la prueba de referencia hasta el momento, estandarizada, de fácil ejecución, bajo costo y disponible. Se consideran valores positivos de absorbancia mayores o iguales a 0,34 , con sensibilidad del $95,7 \%$ y especificidad del $100 \%$. Mediante esta prueba se logra el diagnóstico diferencial de absceso hepático entre amebiano y piógeno. Cuando la ELISA IgG contra E. histolytica es negativa, es necesaria la punción del drenaje para precisar la etiología bacteriana, micótica, por otros parásitos.

Los hallazgos y limitaciones de las pruebas serológicas continúan en discusión, los sueros de pacientes con colitis amebiana y AHA han mostrado valores altos de anticuerpos. Dada la persistencia de títulos positivos en áreas endémicas por años, es conveniente correlacionar el cuadro clínico con el resultado de esta prueba; IgG contra E. Histolytica. Sin el contexto de la historia clínica, no permite diferenciar una infección aguda de una pasada, así, frente a un resultado positivo por ELISA en un paciente con absceso hepático (AH) que no mejora al tratamiento con metronidazol, al menos después de 72 horas, se debe realizar punción hepática dirigida por ecografía y proceder a realizar todos los exámenes descritos anteriormente.

Se debe realizar punción dirigida por ultrasonido en las siguientes circunstancias:

- Si el paciente muestra una mala respuesta clínica a la administración de amebicidas transcurridas las 72 horas de tratamiento.

- Cercanía con la vena cava inferior.

- Pericardio y peritoneo.

- Serología negativa para la E. histolytica. 


\section{Diagnóstico diferencial del absceso hepático amebiano}

Vol. 3, núm. 4., (2019)

Alex Christopher Ruiz Arriciaga; Katherine Sofía Méndez Pereira;

Jean Carlos Torres Fernández

Otras indicaciones si se sospecha de otras etiologías, si la ruptura del absceso es inminente o en caso de lesiones grandes o de abscesos localizados en lóbulo izquierdo. La última palabra la tendrá el médico tratante teniendo en consideración posibles contraindicaciones de la posible punción: ascitis, diátesis hemorrágicas o inexperiencia del operador (Rico, 2017).

\section{Tratamiento.}

El fármaco más utilizado para tratar el absceso hepático amebiano es el metronidazol 500 a $750 \mathrm{mg}$ por vía oral o intravenosa, tres veces al día durante 7 a 10 días (30 a 50 mg / Kg/ día vía oral; y de $7.5 \mathrm{mg} / \mathrm{Kg} /$ dosis vía endovenosa). La vía endovenosa no ofrece ninguna ventaja significativa, siempre y cuando el paciente pueda tomar medicamentos por vía oral y no tenga deficiencias en la absorción del intestino delgado. Las tasas de curación son 95\% con desaparición de la fiebre, el dolor y la anorexia entre las 72 y 96 horas. Posterior al tratamiento con metronidazol debe administrarse un fármaco luminal para erradicar el estado de portador asintomático (Centro Nacional de Excelencia Tecnológica en Salud, 2013).

En presencia de intolerancia a la ingesta de metronidazol, se deberá utilizar tinidazol u ornidazol a una dosis de 60 mg por kilo/ día, máximo 2 grs. por diez días; Se siguiere la ingesta de alimentos ricos en hierro en conjunto con el tratamiento con imidazoles. Se puede utilizar nitazoxanida cuando existe intolerancia a los imidazoles. La aspiración terapéutica además del metronidazol para acelerar la resolución clínica o radiológica de los abscesos hepáticos amebianos sin complicaciones no puede apoyarse o refutarse con las evidencias actuales. Los pacientes que no responden adecuadamente al metronidazol o tienen recaídas al tratamiento, deberán ser 


\section{Diagnóstico diferencial del absceso hepático amebiano}

Vol. 3, núm. 4., (2019)

Alex Christopher Ruiz Arriciaga; Katherine Sofía Méndez Pereira;

Jean Carlos Torres Fernández

sometidos a punción percutánea e identificación de la ameba, si se confirma, se sugerirá prolongar el tratamiento con metronidazol.

Se iniciará tratamiento ante la sospecha de absceso hepático amebiano, se indicará punción percutánea si el paciente presenta: persistencia de síntomas clínicos como dolor y fiebre, datos de ruptura inminente de absceso, absceso de lóbulo hepático izquierdo, mujeres embarazadas y contraindicación del uso del metronidazol, complicaciones pleuro pulmonares, paciente sin mejoría después de 72 horas de haber iniciado el tratamiento.

El drenaje laparoscópico combinado con antibiótico terapia es una alternativa quirúrgica en paciente seleccionado o posterior a fracaso de drenaje percutáneo. Dejando la laparotomía solo para casos en los que se sospeche ruptura del absceso a la cavidad peritoneal o no se cuente con los recursos para punción percutánea o cirugía laparoscópica.

\section{Conclusión.}

La amibiasis intestinal y extra intestinal sigue siendo un problema de salud pública en países de área tropical; debe ser tenida en consideración para cuadros clínicos abdominales, ante la presencia de absceso hepático. La etiología más frecuente de absceso hepático sigue siendo la amebiana. Los resultados de laboratorio en el contexto de la historia clínica y los aspectos epidemiológicos son claves para un diagnóstico preciso. Una opción efectiva para el diagnóstico se realiza mediante la prueba de ELISA IgG contra E. Histolytica, es la prueba de referencia hasta el momento, estandarizada, de fácil ejecución, bajo costo y disponible. Mediante esta prueba se logra el diagnóstico diferencial de absceso hepático entre amebiano y piógeno. Cuando la ELISA 


\section{Diagnóstico diferencial del absceso hepático amebiano}

Vol. 3, núm. 4., (2019)

Alex Christopher Ruiz Arriciaga; Katherine Sofía Méndez Pereira;

Jean Carlos Torres Fernández

IgG contra E. histolytica es negativa, es necesaria la punción del drenaje para precisar la etiología bacteriana, micótica, por otros parásitos.

La estudios de endoscopia de vías digestivas bajas se emplean para visualizar la lesión y hacer el diagnóstico diferencias con otras patologías del colon como la colitis ulcerativa mediante el estudio histopatológico. Para la amebiasis extra intestinal, el cuadro clínico más frecuente es el absceso hepático para el cual se parte del cuadro clínico, seguido por la ecografía hepática y el inmunodiagnóstico. La radiografía del tórax permite precisar el compromiso pleural, pulmonar o pericárdico.

El fármaco más utilizado para tratar el absceso hepático amebiano es el metronidazol 500 a $750 \mathrm{mg}$ por vía oral o intravenosa, en presencia de intolerancia a la ingesta de metronidazol, se deberá utilizar tinidazol u ornidazol. Se sugiere la ingesta de alimentos ricos en hierro en conjunto con el tratamiento con imidazoles. Se puede utilizar nitazoxanida cuando existe intolerancia a los imidazoles. La aspiración terapéutica además del metronidazol para acelerar la resolución clínica o radiológica de los abscesos hepáticos amebianos. Los pacientes que no responden adecuadamente al metronidazol o tienen recaídas al tratamiento, deberán ser sometidos a punción percutánea e identificación de la ameba, si se confirma, se sugerirá prolongar el tratamiento con metronidazol.

El absceso hepático amebiano es una patología relacionada directamente con las malas condiciones sanitarias, su incidencia disminuye con medidas que tiendan a mejorar estas condiciones. No todos los infectados progresan a AHA o hay casos de AHA sin colitis amebiana 


\section{Diagnóstico diferencial del absceso hepático amebiano}

Vol. 3, núm. 4., (2019)

Alex Christopher Ruiz Arriciaga; Katherine Sofía Méndez Pereira;

Jean Carlos Torres Fernández

previa o concomitante. En un país con alta incidencia de amibiasis la complicación hepática es relativamente frecuente.

\section{Bibliografía.}

Bernejo, J. (Junio de 2014). Tratamiento del absceso hepatico. experencia en el Hospital Nacional de Itaugua. Cirugia paraguaya, 38(2), 1-13. Obtenido de Hospital Nacional de Itagua.

Centro Nacional de Excelencia Tecnológica en Salud. (2013). Absceso hepático amebiano. No complicado. Obtenido de Centro Nacional de Excelencia Tecnológica en Salud (CENETEC): https://www.elsevier.es

Feldman, M. (18 de Mayo de 2017). Absceso hepático amebiano. Obtenido de American Accreditation HealthCare Commission (ADAM): http: www.eclinicalworks.adam.com

Girón, W. (Diciembre 2011). Absceso hepático amebiano. Revista Facultad Ciencia Médica. Volumen $8, N^{\circ} 2$, http:cidbimena.desastres.hn.

López, M. (2008). Diagnóstico de amebiasis intestinal y extra intestinal. Acta Médica Colombiana Volumen 32, 75 - 83

Marin, E. (Octubre 2000). Absceso hepático amebiano. Acta Médica Colombiana Volumen 25 $N^{\circ} 5,218-226$ http:www.actamedicacolombiana.com.

Pereira, M. (Marzo de 2014). Absceso hepático amebiano. Revista de Cirugía Pediátrica CIRUPED. Vol. 4, No. 1., www.ciruped.org.

Rico, A. (Diciembre de 2017). Complicaciones pulmonares de absceso hepático amebiano. Obtenido de Docsity: http:www.docsity.com

Viasus, D. (Octubre de 2004). Inmunología del absceso hepático amebiano. Obtenido de SciELO - Scientific Electronic Library Online.: http:www.scielosp.org

$$
\text { (c) (1) (9) (2) }
$$

RECONOCIMIENTO-NOCOMERCIAL-COMPARTIRIGUAL

CC BY-NC-SA

ESTA LICENCIA PERMITE A OTROS ENTREMEZCLAR, AJUSTAR Y CONSTRUIR A PARTIR DE SU OBRA CON FINES NO COMERCIALES, SIEMPRE Y CUANDO LE RECONOZCAN LA AUTORÍA Y SUS NUEVAS CREACIONES ESTÉN BAJO UNA LICENCIA CON LOS MISMOS TÉRMINOS. 